\title{
COGNITIVE AND NEUROLOGICAL OUTCOME OF PRETERM INFANTS AT THE AGE OF 5-8 YEARS WITH POST-HAEMORRHAGIC VENTRICULAR DILATATION REQUIRING NEUROSURGICAL INTERVENTION
}

\author{
A.J. Brouwer ${ }^{1}$, C. van $\mathrm{Stam}^{2}$, M. Uniken Venema ${ }^{3}$, C. Koopman ${ }^{4}$, F. Groenendaal ${ }^{4}$, L.S. de Vries ${ }^{5}$ \\ ${ }^{I}$ Neonatology, ${ }^{2}$ Paediatric Psychology, Wilhelmina Children's Hospital, University Medical Centre Utrecht, \\ ${ }^{3}$ Paediatric Psychology, ${ }^{4}$ Neonatology, Wilhelmina Children's Hospital, University Medical Center, Utrecht, \\ ${ }^{5}$ Wilhelmina Children's Hospital, University Medical Center, Utrecht, The Netherlands
}

Background: Post-haemorrhagic ventricular dilatation is not necessarily associated with an unfavourable outcome. Preterm infants who develop progressive PHVD in the absence of associated parenchymal lesions may have a normal neurodevelopmental outcome.

Objectives: To evaluate neurodevelopmental and cognitive outcomes among preterm infants with severe $\mathrm{IVH}$, requiring neurosurgical intervention for their associated PHVD.

Methods: 32 preterm infants with a gestational age (GA) between 26 and 35 weeks were admitted to a neonatal intensive care unit with PHVD requiring neurosurgical intervention, either a ventricular reservoir and/or a ventriculo-peritoneal shunt. All 32 infants were seen in the follow-up clinic and had standardized cognitive, behavioural and neurological assessments between 5 and 8 years of age. For the 23 infants with a $\mathrm{GA}<30$ weeks, matched controls were available and their outcome was compared with the IVH group.

Results: The majority (59.4\%) had no impairments. None of the children with a grade III and eight of the 15 children $(53 \%)$ with a grade IV haemorrhage developed cerebral palsy. More subtle motor problems assessed with the Movement-ABC score were seen in $39 \%(n=9)$, six of whom had an IVH grade III; the mean IQ of all children was 93.4 , and $29 \%$ of the children had an IQ $<85$ (-1SD). Timing of intervention did not have a beneficial effect on neurodevelopmental outcome. With respect to cognition, no significant differences were found between the IVH group and the control group.

Conclusion: Overall, children with a severe haemorrhage had a more favourable outcome than reported previously. 